\title{
Delay Tolerant Lazy Release Consistency for Distributed Shared Memory in Opportunistic Networks
}

\author{
Chance Eary, Mohan Kumar*, Gergely Zaruba \\ Department of Computer Science and Engineering, The University of Texas at Arlington, \\ Box 19015, 500 UTA Boulevard, Room ERB 559, Arlington, TX 76019, United States \\ *Department of Computer Science, The Rochester Institute of Technology, 102 Lomb \\ Memorial Dr., Rochester, NY 14623, United States
}

\begin{abstract}
Opportunistic networks (ONs) allow mobile wireless devices to interact with one another through a series of opportunistic contacts. While ONs exploit mobility of devices to route messages and distribute information, the intermittent connections among devices make many traditional computer collaboration paradigms, such as distributed shared memory (DSM), very difficult to realize. DSM systems, developed for traditional networks, rely on relatively stable, consistent connections among participating nodes to function properly.

We propose a novel delay tolerant lazy release consistency (DTLRC) mechanism for implementing distributed shared memory in opportunistic networks. DTLRC permits mobile devices to remain independently productive while separated, and provides a mechanism for nodes to regain coherence of shared memory if and when they meet again. DTLRC allows applications to utilize the most coherent data available, even in the challenged environments typical to opportunistic networks. Simulations demonstrate that DTLRC is a viable concept for enhancing cooperation among mobile wireless devices in opportunistic networking environment.
\end{abstract}

Keywords: Opportunistic Networks, Delay Tolerant Networks, Distributed Systems, Distributed Shared Memory, Lazy Release Consistency

\section{Introduction}

The opportunistic networking paradigm allows mobile wireless devices to interact when they are within communication range of one another [1] [2]. As devices move about their environment and enter one another's transmission

Email addresses: chance.eary@mavs.uta.edu (Chance Eary), mjk@cs.rit.edu (Mohan Kumar*), zaruba@uta.edu (Gergely Zaruba)

Preprint submitted to Elsevier

February 16, 2015

(C) 2015. This manuscript version is made available under the Elsevier user license http://www.elsevier.com/open-access/userlicense/1.0/ 
range, they can create a temporary, point-to-point connection between themselves. This connection presents an opportunity for processes to exchange data and collaborate with no dependence on pre-existing networking infrastructure, or foreknowledge of where a device will be and when.

Because wireless devices in an opportunistic network are mobile, connections among them are erratic and susceptible to being dropped without warning [3]. Devices are assumed to have no ability to determine when, or if, they will again meet to resume collaboration.

Many technologies developed to facilitate collaboration among processes over network connections, such as distributed shared memory (DSM), were intended for use with consistent and stable links between nodes [4]. DSM has been a research topic within computer science for several decades, with the initial implementation being proposed in [5]. Central to all models proposed in traditional distributed computing is that each process participating in the system will have consistent access to shared memory, be it located on the same physical machine or available across a network connection. As DSM systems evolved, different methods to ensure the consistency of shared data were proposed [6]. These schemes began to incorporate mechanisms to increase a system's tolerance to network delay, but still assumed participating nodes and their data would ultimately be accessible when necessary. Within an ON, neither of the aforementioned assumptions are applicable.

To facilitate the collaboration of mobile wireless nodes in the presence of unpredictable and intermittent network connections, we propose delay tolerant lazy release consistency (DTLRC). DTLRC has two goals:

- Allow two or more processes to share content in an $\mathrm{ON}$; and,

- Ensure that a node can continue working on shared data even if it is separated from its peers for extended periods of time.

Using DTLRC as a foundation, we propose Social Cache (SC). Social Cache allows frequently encountering nodes to have increased shared memory.

We carry out extensive simulation studies to evaluate DTLRC. Through various scenarios, we demonstrate that maintaining consistency of shared memory among nodes utilizing brief opportunistic connections is possible.

Section 2 gives an introduction to opportunistic networking and a discussion of existing DSM systems. Section 3 provides an overview of the theory and algorithms used in DTLRC. Section 4 describes the operation of DTLRC. Section 5 discusses the theory and algorithms used in Social Cache. Sections 6 and 7 discuss simulations and analysis of DTLRC. Sections 8 and 9 discuss simulation and analysis of Social Cache. And Section 10 includes closing remarks.

\section{Background}

This section discusses opportunistic networks as well as extant consistency schemes for distributed shared memory in traditional networks. 


\subsection{Opportunistic Networks}

An ON is created by a series of pair-wise opportunistic contacts between devices, distributed in space and time. ONs show great potential in exploiting the mobility of wireless devices by allowing communication with one another when they are in range. ONs require neither infrastructure support not prior planning [1] [2] [3] [7].

When attempting to apply computing techniques intended for traditional wired networks over opportunistic networks, a number of key challenges are encountered. Amongst the most difficult to address is the issue of devices not knowing with any certainty how long other nodes within the $\mathrm{ON}$ will be available to participate in the network. Uncertainties about a node's availability, join and leave patterns, and the length of time for which it remains connected, are major challenges of ONs [1] [2]. This, combined with the intermittent delays and data losses that are typical of the wireless medium [8], makes ONs a highly challenged networking paradigm.

\subsection{Distributed Shared Memory}

One method for executing processes to collaborate with one another over networks is distributed shared memory (DSM). Distributed shared memory allows multiple processes, either on the same physical system or connected via a network, to concurrently operate on a set of data [4]. In order to facilitate content sharing among mobile devices connected opportunistically, an implementation of DSM for use in ONs should be formulated.

Traditional DSM systems are designed for use over networks with relatively high reliability links among highly available nodes [9]. While traditional DSM systems are tolerant to network delays, they were not designed for repeated loss of connections a DSM implementation in ONs would entail. The separated nodes will remain fully functional while disconnected from one another, but their processing power cannot be utilized until the resumption of connectivity. Such disconnections are expected to be commonplace in opportunistic networks.

In order for distributed shared memory to work correctly, all processes participating in the system must have a consistent view of shared data. Consistency schemes are methods that allow processes to decide which value in the shared data is appropriate to use, and which value should be overwritten to reflect more relevant writes. Simply using the "most recent" write at all processes is not feasible, as it is assumed nodes do not share a common clock due to complications from inconsistent network conditions [10]. Many consistency schemes have been proposed over the years [5] [6] [11]. One scheme focusing specifically on memory consistency in potentially high-latency networking environments is lazy release consistency (LRC) [12] [13].

\subsection{Lazy Release Consistency}

LRC utilizes a variety of techniques to avoid synchronizing memory between two processes until absolutely necessary, and then only by exchanging a minimum of data. One such technique is only exchanging consistency information 
between the process releasing its lock on shared data and the process subsequently acquiring that lock. Other participating processes will not update their view of shared memory until they acquire the lock and obtain exclusive access to write to shared memory. This effectively means that some processes' view of shared memory will be allowed to temporarily diverge until they have a need to write to shared memory.

Some systems, such as [14], designed to work in delay tolerant environments have used LRC as their underlying consistency mechanism. However, LRC ultimately assumes that a process and its data will be available when needed.

\subsection{Delay Tolerant Lazy Release Consistency}

Delay tolerant lazy release consistency expands upon core concepts within LRC to create a consistency protocol applicable in opportunistic networks, where loss of end-to-end connectivity between participating processes is common. An example of its employment is discussed and analyzed in $\S 6.1$. The next section discusses the theory and architecture of DTLRC.

\section{Architecture}

Within an opportunistic network, where connections between devices are often fleeting, existing consistency schemes will be relegated to operating only when an end-to-end connection is available.

\subsection{Setup}

When two or more nodes agree to collaborate via DTLRC, they first set aside an area of memory for use in the system. The memory is uniformly divided into segments, with each segment assigned an index. The arrangement of segments and indices is identical for all participating devices, similar to that in [13]. The entire data set necessary for operation is then copied to each machine and stored in the designated area of memory.

Copying the entire shared memory set to a node is a departure from extant schemes. While this creates high overhead during initialization, it permits nodes to work on the dataset regardless of network conditions, as well as communicate changes to the dataset with a minimum of information.

\subsection{Data Races}

While peers in a network maintain a stable connection, an extant scheme of DSM, such as LRC, can be used to maintain consistency and avoid data races. When nodes disconnect from the network, DTLRC does not attempt to avoid data races or establish an ordering of operations between nodes upon the resumption of network connectivity. Rather, DTLRC provides a mechanism to establish a consistent view of memory when a disconnected node regains contact with its peers. Data races are accepted when nodes are disconnected and acting independently. 
As nodes are assumed to have no ability to coordinate in the absence of a network connection, operations performed on a segment of memory at one node may have no relevance to the comparable segment of memory hosted at another node. Therefore, attempting to establish an ordering of operations between two nodes acting independently would not result in a meaningful state of memory. Because of this, DTLRC selects the most appropriate value written by either node to be shared between the peers during an opportunistic contact. In this sense, a write conflict is not a competing order of operations, but the contents of an index in shared memory that is not identical between two or more nodes.

\subsection{Memory Metadata}

Metadata for each index comprises three fields:

- Cluster ID: if contiguous segments of shared memory are related to one another, they are assigned to a cluster. Clusters allow portions of memory to be written to atomically;

- Priority: should an application determine that a write is of high importance, an elevated priority can be assigned to this write. Priority is represented as an integer with the maximum priority set according to the requirements of the application; and,

- Write history: contains three subfields -

- Origin: the node ID that created this write;

- Time of creation or creation time: the internal time at the origin node when this write was created; and,

- Time of receipt or received time: the time at the local node when this value was received. Received time will be equal to creation time if the value was written locally.

During an opportunistic contact, these metadata values are encapsulated into objects called diffs and used by the meeting nodes to communicate changes to memory.

\subsection{Diffs}

Information about operations to memory can be communicated via diffs, as memory is identically arranged on all participating nodes. Diffs, conceptually similar to those used in [13], are objects recording the represented index and containing the metadata for that index in shared memory. A diff does not contain the actual value to be exchanged between nodes, just the associated metadata. The diffs are exchanged between two previously separated nodes to determine the most appropriate way to apply writes to shared memory, as described in $\S 3.6$.

Figure 1 provides an example of metadata. Here at node 'A', index 21 has been assigned to cluster 3 , with no elevated priority. This write object was 


\begin{tabular}{|c|c|c|c|c|c|}
\hline Index & Cluster & Priority & History & \multicolumn{2}{|c|}{} \\
\hline 21 & 3 & 0 & Origin & Creation & Received \\
\hline & & B & 1776 & 2012 \\
\cline { 5 - 6 } & & & & &
\end{tabular}

Figure 1: Example metadata for index 21 at node 'A'

created at node 'B' at time ' 1776 ' and received at node 'A' at time '2012'. In the event of node ' $\mathrm{A}$ ' receiving write objects for index 21 from nodes other than ' $\mathrm{B}$ ', additional entries in the write history would be included. The history for the current write at node ' $\mathrm{A}$ ' is sorted to the first position in the write history. These data are included in the diffs created by node ' $\mathrm{A}$ ' when it makes an opportunistic contact.

\subsection{Write Conflicts}

While a node is operating on its own, it is free to read and write to its copy of shared memory whenever required by the process. When a node makes a modification to shared memory, it stores the metadata for its write in its own write history. Any node with which this process was collaborating prior to being disconnected can also read and write freely to their copies of shared memory.

Because these nodes are unable to communicate in the absence of a network connection, two or more nodes may write to the same index in their local copy of shared memory, creating a write conflict. If nodes carrying a copy of shared memory do not encounter one another again, these write conflicts are irrelevant and can be ignored; the system will continue working on its copy of memory, with the only additional overhead being the updates to the shared memory metadata. When nodes carrying shared memory do meet one another later, they will negotiate which writes to retain in the conflict resolution phase, as explained below.

\subsection{Conflict Resolution}

DTLRC assumes no global clock (such as that provided by a global positioning system or cellular phone network). As writes cannot directly affect one another while nodes have no end-to-end network connection, simply determining event order is unproductive, even if a global clock were available.

A write produced by a process is automatically selected for retention if there are no conflicts. When a write conflict does occur, three methods are used for selecting the most appropriate write to retain. They are:

- Priority-based: If two writes to an index have different priorities, the write with the highest priority is selected for retention; 
- Cluster-based: Writes to a cluster at two different nodes conflict as the segments in a cluster are directly related and should be updated atomically. The resolution protocol will select writes to this cluster from one of the nodes for retention; and,

- Volume-based: Should the preceding criteria be equal or otherwise not applicable, the node that has produced the highest volume of writes will have its write retained in the event of a conflict.

By applying the conflict resolution protocols, nodes can agree on a consistent view of memory. While only two nodes will synchronize at a time [13], those nodes can then propagate their agreed upon view of memory to other nodes. As opportunistic contacts continue to occur, processes continue synchronizing the contents of their local copy of shared memory while assimilating new writes into the system.

\subsection{Write History}

In ONs, two or more nodes can independently write to the same index and share the written values with other nodes. As these written values propagate through the network, a receiving node may repeatedly encounter different values for the same index. This leads to a condition called a 'historical conflict', where the current value of an index at a receiving node may be overwritten by a previously encountered value that node has overwritten in the past. To prevent nodes from having values repeatedly overwritten, a write history is kept for each index.

\subsubsection{Updating Write History}

When an index is updated, the following values are recorded: the ID of the originating node; the local time at the origin node where the write was created; and the time when the write object was received at the local node. As nodes synchronize their local copies of memory, they check incoming diffs against the write history for the affected index. If the same values for origin ID and creation time are found in the write history, the node knows it has seen this value previously. The conflict resolution protocol would then select the node's local value to be applied to the shared memory of the remote process.

\subsubsection{Historical Synchronization}

Synchronizing nodes create a write history for both the value that was retained, as well as the value that has been overwritten. Entries in the write history can be removed when their received time exceeds the notification interval, as discussed in §3.7.4. This is sufficient to ensure values, which have previously been selected to overwrite, are eventually removed from the local cache of all nodes. 


\subsubsection{Timestamps}

It is important to note that the timestamp maintained within the write history is only used as an indication of chronology when comparing later write objects originating from the same node to the same index. If a later write object to an index is seen from the same node, its existing entry in the write history is updated.

Timestamps from two different nodes are only compared to one another in the special circumstance that each node has previously seen the other's current write in a segment. In this case, the write object with the 'most recent' creation timestamp is selected for retention. The value of these timestamps is not assumed to be an accurate indicator of event order: the values are used because the resolution will be the same anywhere this conflict occurs in the network.

\subsubsection{Notification Interval}

The received time field is used to determine the length of time the node should notify its peers that the value in this index needs to be shared. After this period of time, the metadata for that write will be removed from the write history.

Based on the rate of contacts between nodes in the system per unit time, DTLRC can select a 'notification interval' (NI). The NI is set as the estimated amount of time it would take a single write object from a single node to propagate to a percentage of the number of nodes participating in the system, if there were no write conflicts. As data begins to propagate more readily, either due to an increase in the number of participant nodes or an increase in the internode contact rate, the NI decreases and vice versa. When the NI decreases, the upper bound on the necessary metadata is tightened. The NI is continuously readjusted based on the contact patterns in the network. Should the amount of memory dedicated to the write history become imprudent, the NI can be manually shortened to expunge additional metadata from the system and reduce memory overhead.

During an opportunistic contact, the initiating process examines memory to determine which writes have been received or created within the notification interval, and selects those writes for further consideration by its peers by sharing diffs for those indices. Without the NI, nodes would continue to notify one another about every memory update that has taken place since the instantiation of the system.

\subsubsection{Flushing Diffs}

In ONs, it is possible for a node to be separated from its peers for the entirety of the notification interval. Any writes generated by such a node during this period would ultimately be lost to the system, as the node will not generate diffs for these writes when it again encounters its peers. Due to the system not creating diffs for these writes, this data will not be shared with peers. To address this, a node can 'flush' its diffs if a separation exceeds the length of the notification interval. 
When a node flushes its diffs, any write that was created since the last time this node encountered one of its peers will have a diff created for it. This ensures that a node experiencing an abnormally long loss of connectivity with its peers will still have its updated data considered for retention by the system.

\subsection{Overhead}

A primary generator of activity in memory is the rate of opportunistic contacts occurring within the network. A higher number of contacts means more

data is shared between nodes, resulting in the runtime-overhead necessarily increasing. The mechanism providing an upper-bound on the runtime overhead is the notification interval.

As the rate of opportunistic contacts increases, the NI decreases, as discussed in §3.7.4. As the NI decreases, the overhead created by storing and maintaining the write history remains bounded, as entries in the write history are released with greater frequency.

Furthermore, an increase in the number of nodes participating in the system does not result in more memory being required by each node. While the contents of memory will diverge, the arrangement and size of shared memory dedicated to the system is fixed and identical on every participating node, as described in $\S 3.1$.

The next section discusses the operation of DTLRC.

\section{Operation}

This section describes the procedure followed when a node utilizing DTLRC experiences an opportunistic contact. Figure 2 illustrates the steps in the conflict resolution protocol.

\subsection{Acquiring and Releasing Locks}

DTLRC does not use locks to maintain consistency to shared data, however the concept is implicitly integrated due to the nature of connections within an opportunistic network. Because nodes cannot exchange consistency information while separated they have exclusive access to their copy of shared memory, effectively "locking" that data. During an opportunistic contact, the host processes of two nodes, A and B, have the ability to exchange consistency data. This allows A to write to B's copy of shared memory, and vice versa, effectively "releasing" the locks on A and B's respective datasets.

Consistency information is only shared between two processes at a time. Copies of shared data pertaining to other processes are allowed to temporarily diverge until their host node's next opportunistic contact. This is conceptually similar to the operation of lazy release consistency, as discussed in $\S 2.3$. 


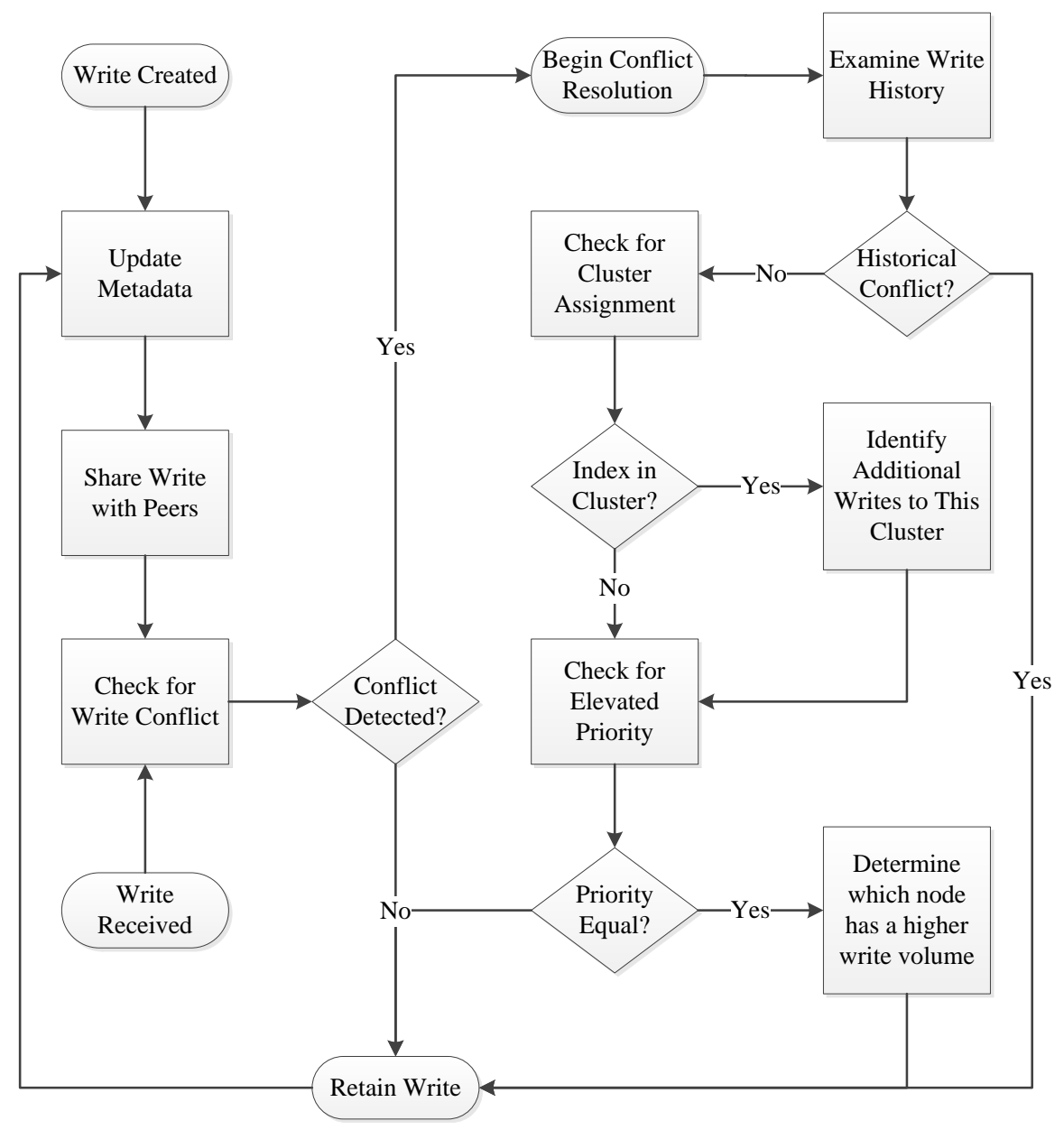

Figure 2: Progression of Conflict Resolution Protocol

\subsection{Diff List}

When two collaborating nodes have an opportunistic contact, they examine the metadata of their local copy of shared memory. If the received time for an index is within the notification interval, a diff is created for this index and added to a list. The resulting list of diffs, or diff list, is sent to the node that initiated the contact. The initiating node prepares its own diff list to compare against the received diff list. 


\subsection{Identifying Write Conflicts}

Due to memory being identically configured on each node, any index present in the diff lists of both nodes indicates a write conflict has occurred. If an index is present in one diff list but not the other, this represents an uncontested write, and can safely be applied to the shared memory of both nodes. Once conflicts are identified, the initiating node can then use the included metadata to autonomously resolve write conflicts in a meaningful manner. The first step in the resolution process is resolving historical conflicts.

\subsection{Resolving Historical Conflicts}

To prevent a write currently selected for retention by the system from being overwritten by a write previously selected for removal, the write history for this diff is examined. If the most recent entry in the write history of the conflicting diffs is the same, both nodes currently share the same write; this write object was simply received within the notification interval and thusly had a diff created for it. If the writes are not the same, the initiating node will attempt to find the most recent write to this index in the write history of the remote node. Three outcomes are possible:

1. Both nodes have previously seen the other's current write. Here, the write with the greatest creation time is selected for retention. Again, it is important to note that the greatest creation time is not an accurate indication of chronology. The creation time is simply a static value that will be associated with this write for the duration of its existence in the system;

2. One node has seen the other's current write, but not vice versa. The previously encountered write will be overwritten; or,

3. Neither node has seen the other's current write. This write conflict will be resolved with another protocol.

If the write conflict was resolved by historical resolution, the system moves on to examine other diffs. If the write conflict was not resolved, the system uses one of the conflict resolution protocols described in $\S 3.6$.

\subsection{Conflict Resolution Order of Operations}

After historical conflicts are resolved, DTLRC resolves remaining conflicts using the above mentioned protocols in the following order: 1) priority-based, 2) cluster-based and 3) volume-based. This order of operations will produce a useful set of data, however the order of the resolution protocols could be adjusted with a simple API switch, should a developer feel an alternative series would be more appropriate. 


\subsection{Retaining Writes}

As the initiating nodes resolves conflicts between the two diff lists, the diffs of writes that have been selected to share are added to a 'retained' diff list. Once the conflict resolution process has completed, the initiating node exchanges the retained diff list with the remote process, along with its writes that have been selected for retention. The remote process responds with its writes that have been selected for retention.

Both nodes then proceed to update shared memory with the newly received values, as well as updating the shared memory metadata and write history at the updated segments. Writes are applied to memory as they are received, with the exception of writes to a cluster: all writes to a cluster must be received at a node prior to applying them to memory, such that if two nodes are disconnected while updating memory, the cluster is not left in an indeterminate state.

The next section discusses Social Cache, which leverages and expands upon DTLRC for groups of nodes that repeatedly interact.

\section{Social Cache}

If a collection of nodes has a high probability of regularly encountering one another, they can elect to employ Social Cache. Social Cache (SC) is an expansion of DTLRC wherein a group of nodes have access to the entire contents of shared memory amongst all participating nodes. The total capacity of memory available to all systems is perceived to be increased. Each node contributes to the shared memory distributed among peers. SC is similar to traditional

distributed shared memory, while making use of the delay tolerant features of DTLRC.

\subsection{Sharing Memory}

Data objects in SC have indices as in DTLRC. Each member of the system assumes responsibility for a range of indices and the contents of memory therein. For example, objects in a node $n_{i}$ may be assigned indices $i(k) \ldots i(k+1)-1$ where $1 \leq i \leq N, N$ is the number of nodes, and $k$ is the number of indices per node.

Nodes within SC are the owners of the data assigned to their range of indices. When another node wishes to access that data, it first determines which node in the system is responsible for that data and enters a request into a local queue. Upon meeting the node responsible for this range of memory, a temporary copy of the data is made on the requesting node. Multiple devices can make temporary copies of a piece of data at the same time, and all can read or write to that section of memory freely.

When a node completes modifications to its temporary copy of data, it creates diffs and holds them in a queue. Upon meeting the owner of that data, the diffs are submitted and the temporary copy is dropped from the requesting node. The owner of the data maintains a notification interval based on the expected amount of time it would take to meet every node in the group. Because 
multiple nodes can write to a temporary copy of the data at a time, updates received from other nodes within the notification interval are screened with the DTLRC's conflict resolution protocols, then applied to the data.

\subsection{Advantages and Disadvantages of SC over DTLRC}

As well as increasing the amount of memory available to the system, the arrangement of memory on each system no longer has to be symmetrical. A resource constrained device can allocate the amount of memory it has, while still making use of the memory on the other systems. This arrangement better accommodates a heterogenous collection of computing platforms participating in the network.

While allowing increased flexibility in memory configurations and a greater variety of computing platforms, a delay in requesting and receiving data will be inherent in the operation of SC. The amount of delay that can be tolerated is ultimately defined by the consumer of the data.

Utilizing SC also creates a reliance on the presence of a node in the network. Should any device become permanently disconnected from the system, its shared memory data will be lost. Depending on the degree of participation of the departing node in the network, the distributed shared memory system may cease to function.

\section{DTLRC Evaluation}

This section discusses the simulations conducted to demonstrate the viability of DTLRC and Social Cache. The data traces, scenarios, plots and results are provided below.

\subsection{Simulation Scenario}

The foundation for the simulations is based on an actual semi-annual event that takes place in Fayette County of the US state of Texas called "The Texas Antique Week". This event is typically attended by over 1,000 antiques vendors and upwards of 10,000 customers on its busiest days [15].

The primary fairgrounds extend southwest along Texas State Highway 237 from Round Top, TX to Warrenton, TX - a distance of over six kilometers. Additional fairgrounds are set up between nearby towns, sporadically appearing for another 7 kilometers. Vendors will occupy available land on the side of this two lane road, leaving attendees with a single route into, between, and out of the various fairgrounds [15].

Round Top has a permanent population of 90 people, and Warrenton has no permanent residents [16]. There are no publicly available WiFi connections to the Internet, and cell phone data service is severely impeded due to an influx

of users. This presents a large collection of mobile users with smart phones compacted into a relatively small area, and, with restricted options for transit, who are temporarily isolated from outside data connections. 
In our scenario, dealers compile a spreadsheet of their wares with a description, photo and price. At the event, these spreadsheets are shared via wireless devices operating in ad hoc mode. Using this data, dealers hold a silent auction modeled as a winner determination problem [17].

Users place bids on different combinations of items and share competing bids amongst themselves. At the end of the bidding period, the user with highest bid receives his or her items.

\subsection{Movement Model}

To provide a realistic model for the movement of patrons around the exhibition grounds, the Home-cell Community-based Mobility Model (HCMM)[18] was used. HCMM models both socially-driven mobility as well as location-based mobility [19]. Considering the impact of socially-driven mobility in this specific scenario is important: attendees to the event can be expected to move as a loosely associated group to points of common interest, and no customer is under any obligation to visit every booth at the fair, leaving some parts of the map untraversed. Traces of opportunistic contacts were generated and then fed into a custom simulator that models the behavior of DTLRC.

\subsection{Simulation Configuration}

To model a portion of the fair grounds, as described in $\S 6.1$, HCMM was configured to model users with wireless devices moving around a $500 \mathrm{~m}^{2}$ environment. Each scenario modeled either 10, 25, 50 or 100 users walking on foot. Connections must meet the criteria specified in $\S 6.4$ to qualify as a useful opportunistic contact.

Cells, locations in the simulated map where users congregate [18], were modeled in a 50x50 grid. User's movement speed was set to vary between 1.0 and 3.0 meters per second. Total simulation time was 8 hours.

\subsection{Connection Model}

Bluetooth was used as the basis for connections in these simulations. In Bluetooth, a typical inquiry time is 10.24 seconds, while a typical connection setup between two mutually unknown devices is 5.76 seconds [20]. Bluetooth devices were configured for a connection range of 30 meters and a connection speed of 2.0 Mbps [21].

In the worst case shared memory scenario, devices would need to exchange their entire dataset to a peer who recently entered the network. Given that the devices were configured to share $500 \mathrm{~KB}$ of shared memory, exchanging the full dataset between two nodes would take 2 seconds at a connection speed of 2.0 Mbps. In addition to exchanging the dataset, the simulation assumes that an additional 2 seconds would be needed to exchange the full table of metadata and for each node to identify write conflicts.

Times associated with the connection model are summarized in Table 1.

Based on these values, two nodes in the movement model must be within 30

meters of one another for at least 20 seconds to consider the connection viable for utilization. 
Table 1: Time Summaries

\begin{tabular}{|c|c|}
\hline Parameter & Duration (s) \\
\hline Inquiry & 10.24 \\
Connection Setup & 5.76 \\
Dataset Exchange & 2.0 \\
Metadata Exchange & 2.0 \\
\hline Total & $\mathbf{2 0 . 0}$ \\
\hline
\end{tabular}

\subsection{DTLRC Configuration}

Each device was configured with $500 \mathrm{~KB}$ of shared memory, divided into 62,500 indices, or roughly 8 bytes per index. Writes are uniformly distributed across shared memory, and occur every five to fifteen minutes. Each of the simulated devices share writes with every other device. Each of the shared memory scenarios were run a total of 30 times, and the results of the trials compared here.

\subsection{Comparison to Extant DSM Systems}

Performing an effective comparison between DTLRC and existing systems presents several challenges. As stated previously, opportunistic networks are suitable only for delay tolerant applications. Common performance metrics, such as response time, would therefore not be an appropriate measurement in this environment.

Furthermore, application performance in opportunistic networks is heavily dependent on the connectivity between nodes. Some metrics, such as throughput, would be less indicative of a system's performance, and more a reflection on the underlying mobility patterns of nodes.

In an attempt to provide a one-to-one comparison between DTLRC and similar systems, two variations of DTLRC were simulated. These variations are not being proposed for implementation, but are simulated to provide a simple baseline comparison.

These variations used static nodes, arranged in a basic grid over the simulation area, as depicted in figure 3. A grid layout was used for simplicity, as arranging an optimal circle packing is non-trivial. WiFi was used as the connection medium, with a range of $100 \mathrm{~m}$. Requirements to count as a viable opportunistic contact following specifications outlined in $\S 6.4$. In simulations with static nodes ("static simulations" for brevity), mobile nodes could only communicate with static nodes, and not with other mobile nodes. Static simulations were configured in two modes:

1. Connected: Static nodes were connected to one another and could share data between themselves, as well as mobile nodes; and,

2. Disconnected: Static nodes could only share writes with mobile nodes, and not directly with other static nodes. 


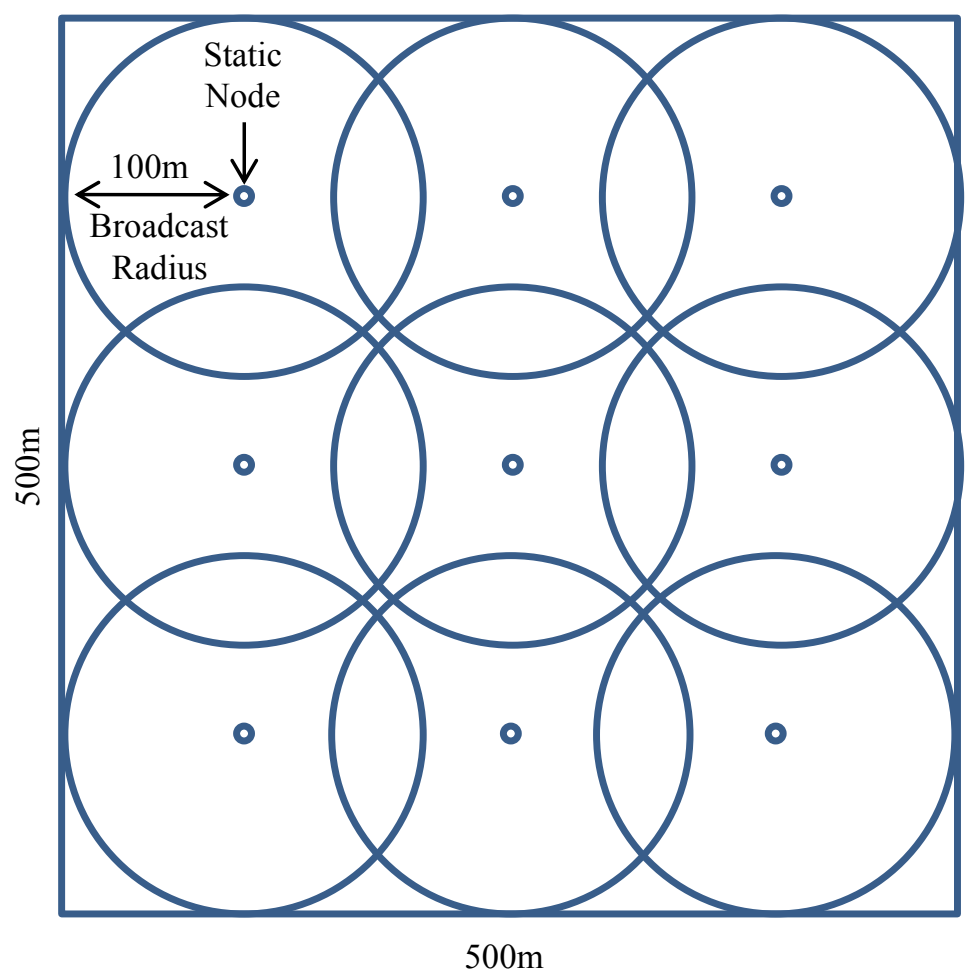

Figure 3: Static Node Simulation Area Layout

The number of static nodes was sequentially incremented from one to nine. Nine nodes became the upper bound as their combined broadcast radii effectively covered the entire simulation area. Due to space requirements, results from varying the number of static nodes have been amalgamated to demonstrate rudimentary trends.

\subsection{Metrics}

To illustrate the performance of DTLRC, the following metrics were recorded in each simulation and analyzed.

\subsubsection{State}

State measures how many different versions of shared memory exist in the system. As processes produce writes to shared memory, the number of different copies of memory in the system will increase. As nodes meet and share their writes, the number of versions decreases as memory is made consistent.

State is divided into three categories: vicinity-based, random sample and overall. 
- Vicinity-based: A comparison of the shared memory of a set of nodes within relatively close physical proximity. This implies the sampled nodes have:

- Recently had an opportunistic contact with one another;

- Are likely to have an opportunistic contact in the near future; or,

- Both.

Vicinity-based is an index-by-index comparison. The number of individual indices that are different is recorded;

- Random sample: A comparison of the shared memory of a set of randomly selected nodes from across the network. Selected nodes may or may not be within one anothers immediate physical vicinity. Like vicinity-based state, random sample is an index-by-index comparison; and,

- Overall state: A count of the number of nodes in the system with a unique state of memory. In this metric, a node is considered to have a unique state of memory when one or more indices are different.

Tracking the state will give insight into the cycles of divergence and convergence of the contents of shared memory.

\subsubsection{Writes Exchanged and Write Conflicts}

To illustrate the total flow of data through the system, as well as the subsequential write conflicts, the following metrics are recorded:

- Writes Exchanged (WE): The average total, average mean and average maximum number of writes exchanged per trial;

- Write Conflicts $(W C)$ : The average total, average maximum and average minimum number of write conflicts per trial; and,

- Time to Convergence (TTC): The interval between the first occurrence of a write conflict at a specific index and the last occurrence of a write conflict at that index. The last occurrence of a write conflict at a specific index indicates one of two things:

- No instance of the conflicting write exists in the shared memory of any node in the network; or,

- The different values for writes at this index never fully converge at all nodes in the network. This is the number of unresolved write conflicts (UWC) per trial.

The average number of unresolved write conflicts, the average mean TTC and the average maximum TTC are recorded per trial.

While the number of writes exchanged can be closely tied to the rate of opportunistic contacts occurring in the network, the results help depict what would be expected from DTLRC's deployment in the real world. 


\section{DTLRC Simulation Results}

Results from the DTLRC simulations are presented here.

\subsection{State Results}

This section discusses the state results, as outlined in $§ 6.7 .1$.

\subsubsection{Vicinity-based Versus Random Sample}

Figures 4 through 11 show the results for both random sampling and vicinitybased sampling. Due to space requirements, the results from sequentially increasing the number of static nodes have been amalgamated to demonstrate overarching trends.

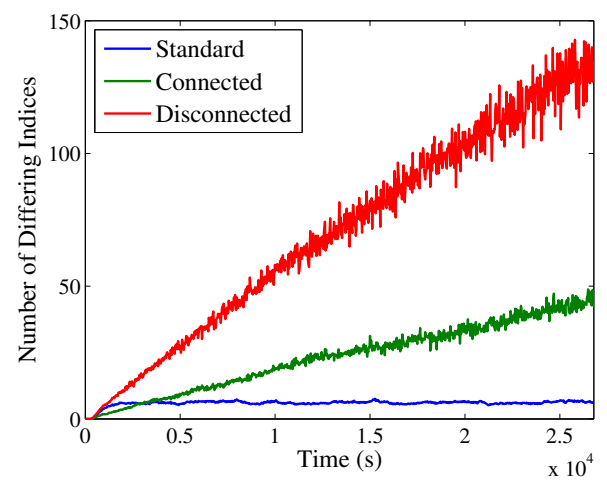

Figure 4: Random Sample: 10 Nodes

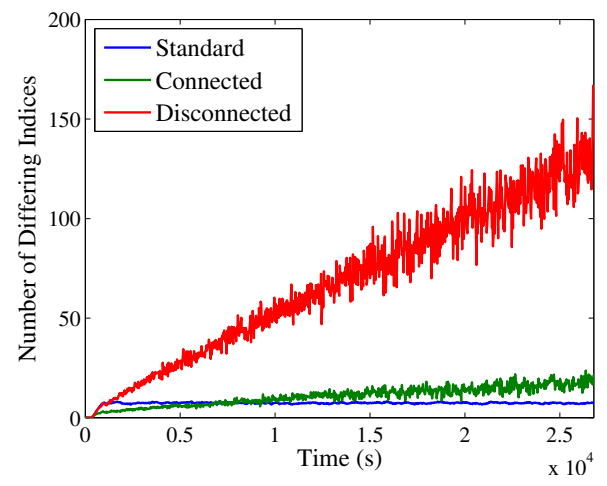

Figure 6: Random Sample: 25 Nodes

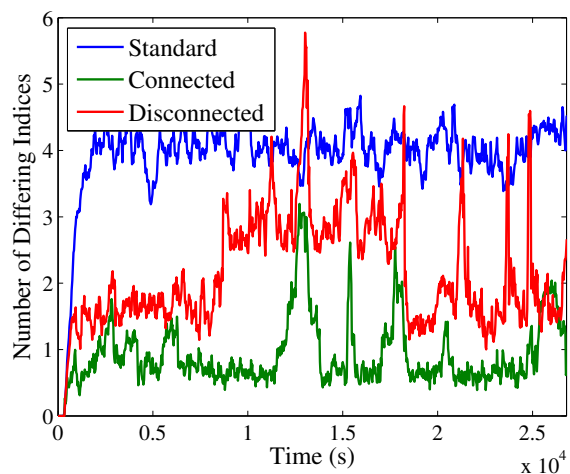

Figure 5: Vicinity-based Sample: 10 Nodes

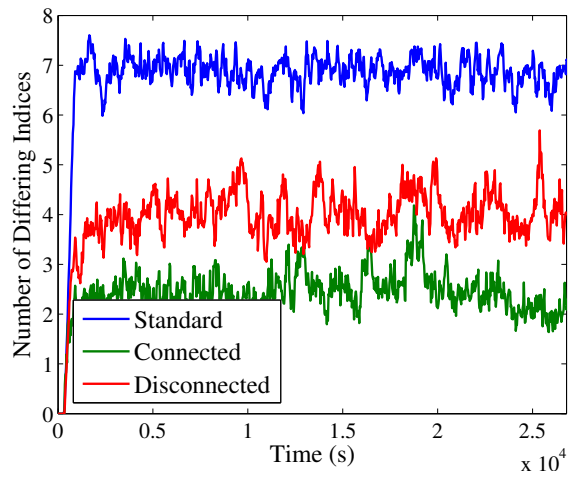

Figure 7: Vicinity-based Sample: 25 Nodes 


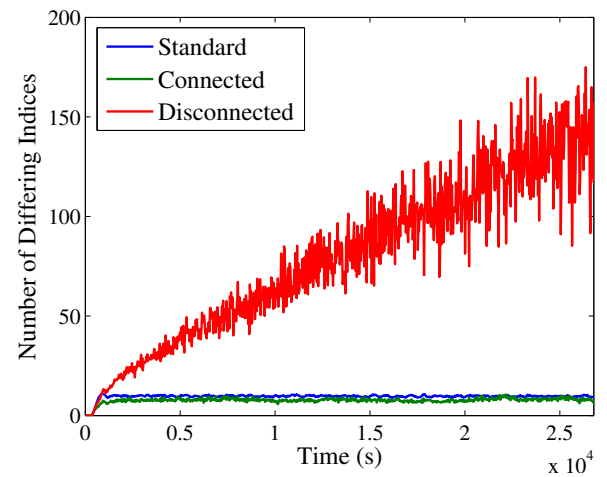

Figure 8: Random Sample: 50 Nodes

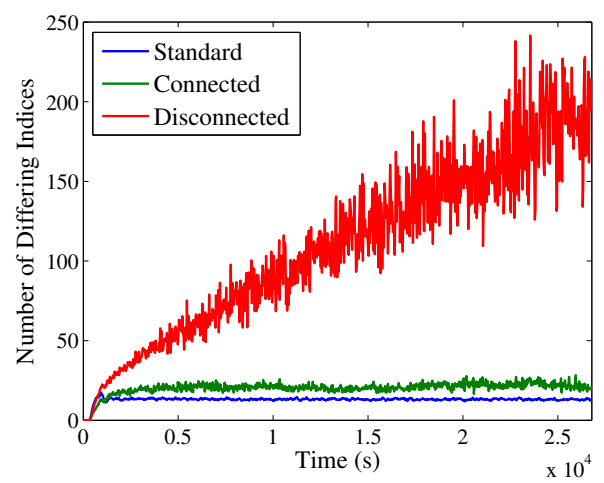

Figure 10: Random Sample: 100 Nodes

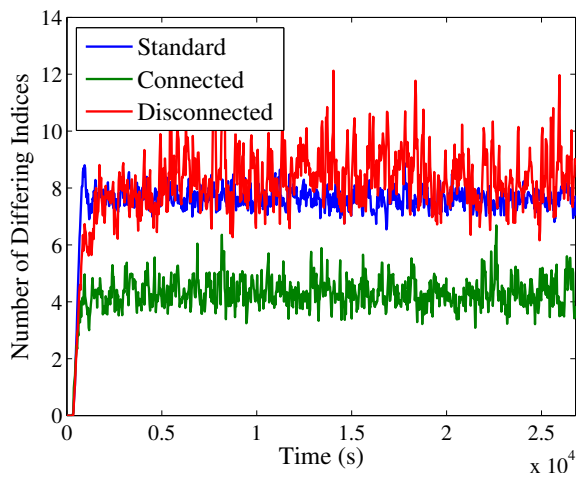

Figure 9: Vicinity-based Sample: 50 Nodes

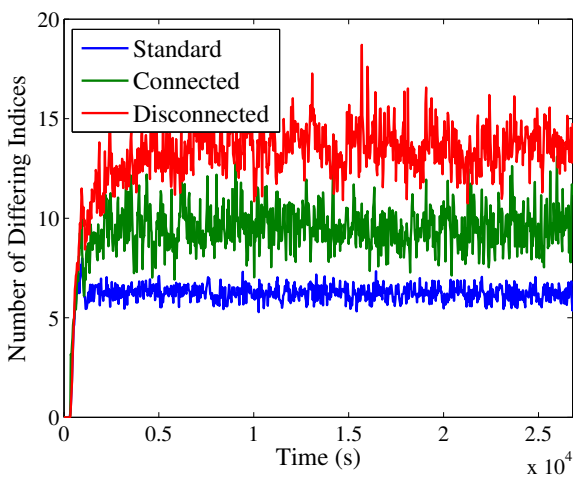

Figure 11: Vicinity-based Sample: 100 Nodes

Across the experiments, connected DTLRC displays a consistently low number of differing memory content between nodes, relative to the other methods. This is to be expected, as connected DTLRC distributes data across a large geographic area without relying on the mobility of nodes to physically move data across the map. However, when there are still a low number of static nodes, connected DTLRC will display steadily increasing memory divergence across the network, unless all mobile nodes move into a static node's transmission range.

The number of differing indices in disconnected DTLRC steadily increases in the random sample, as static nodes cannot share data unless a mobile node carries it to them. As HCMM has no requirement that all nodes move to all parts of the map [18], writes to memory become isolated where mobile nodes travel most often.

In standard DTLRC, the number of differing indices remain relatively consistent throughout all of the trials. The indicated memory divergence of standard DTLRC indicates that the system is scalable in the number of participating nodes it can accommodate, provided those nodes remain in the same general 
area.

\subsubsection{Overall State}

Figures 12 through 14 present the results of the overall state simulations. For these simulations, even a single index of the 62,500 simulated indices being different will result in the node being considered to have a distinct set of memory. The higher the number, the more nodes share identical contents of memory at the sample time.
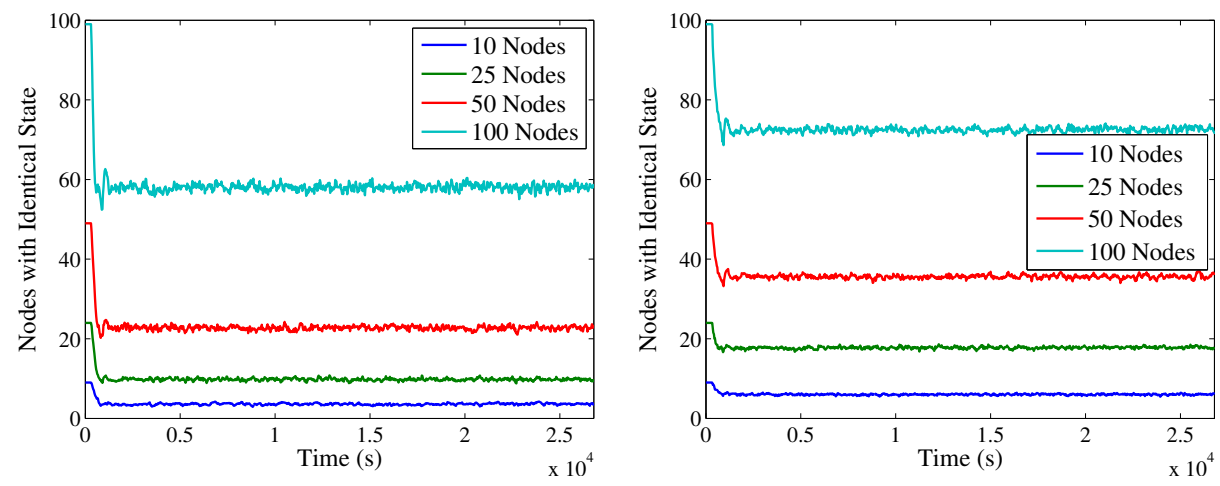

Figure 12: Overall State: Standard DTLRC

Figure 13: Overall State: Connected DTLRC

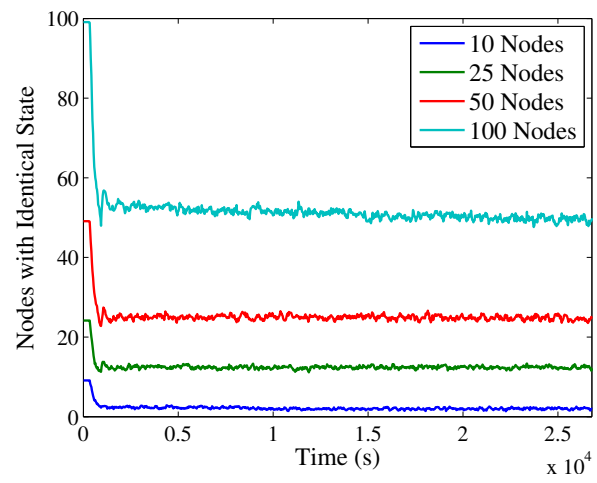

Figure 14: Overall State: Disconnected DTLRC

In the opening seconds of the simulation, all nodes are instantiated with the same contents of memory. This aspect of DTLRC's operation is discussed in $\S 3.1$. After the opening seconds of the simulation, when nodes begin to write to their own copy of memory and share that content with peers, the number of nodes with identical contents of memory drops. Connected DTLRC trends with a higher number of nodes with complete coherence of memory at any sample time. This is because as long as a mobile node is within any static node's 
broadcast radius, its updates to memory will be exchanged to all other nodes within any static node's transmission range.

Disconnected DTLRC results in fewer nodes having identical views of memory, as each static node becomes an isolated pool of data. The static nodes are

dependent on mobile nodes to act as the medium facilitating the exchange of write objects.

For standard DTLRC, the number of nodes with identical contents of memory sit slightly below $50 \%$ in the trials involving 10,25 and 50 nodes. In the 100 node trials, the nodes with exactly the same content of memory sit slightly above $50 \%$. This suggests that the coherence of memory generally increases as the number of participating nodes increases.

\subsection{WE and WC Results}

This section present results of the writes exchanged and write conflict experiments, as outlined in $\S 6.7 .2$. In tables 2 through 9 , results for standard, connected and disconnected configurations of DTLRC are represented by 'S', 'C', and 'D', respectively.

\subsubsection{Writes Exchanged}

Tables 2 and 3 show the number of writes exchanged. In these tables, the "total" is the average number of writes exchanged across all trials. The average "mean" and "max" are per 30 second interval, illustrating the rate at which data are being exchanged between peers.

Table 2: Average Write Exchanges, 10 and 25 Nodes

\begin{tabular}{|c|c|c|c|c|c|c|}
\hline Parameter & \multicolumn{3}{|c|}{$\mathbf{1 0}$} & \multicolumn{3}{|c|}{$\mathbf{2 5}$} \\
\hline & $\mathrm{S}$ & $\mathrm{C}$ & $\mathrm{D}$ & $\mathrm{S}$ & $\mathrm{C}$ & $\mathrm{D}$ \\
\hline Total & 5.1 & 4.6 & 7.5 & 29.2 & 30.9 & 33.4 \\
Mean & 4.2 & 0.7 & 1.9 & 5.3 & 2.3 & 3.2 \\
Max & 4.6 & 1.7 & 4.5 & 10.5 & 6.8 & 9.9 \\
\hline
\end{tabular}

Table 3: Average Write Exchanges, 50 and 100 Nodes

\begin{tabular}{|c|c|c|c|c|c|c|}
\hline Parameter & \multicolumn{3}{|c|}{50} & \multicolumn{3}{c|}{$\mathbf{1 0 0}$} \\
\hline & $\mathrm{S}$ & $\mathrm{C}$ & $\mathrm{D}$ & $\mathrm{S}$ & $\mathrm{C}$ & $\mathrm{D}$ \\
\hline Total & 119.1 & 124.3 & 122.1 & 480.9 & 487.5 & 443.4 \\
Mean & 5.7 & 4.1 & 6.7 & 4.8 & 9.2 & 13.5 \\
Max & 20.6 & 20.6 & 39.4 & 57.1 & 57.6 & 69.2 \\
\hline
\end{tabular}

Disconnected DTLRC presents as having more data being exchanged at any given moment than connected DTLRC, while the total result of data exchanged is comparable to its counterpart connected system. This is because each static node is isolated from every other static node: any update at one static node needs to be independently carried and shared with another static node by a 
mobile node. This results in spikes of data transmission, as a mobile node may need to offload large amounts of data should it move into the range of a geographically isolated static node.

In standard DTLRC, the mean number of writes exchanged per 30 second interval remains relatively consistent through all trials, regardless of the number of mobile nodes. This indicates the network load remains stable, provided a sufficient density of mobile nodes is maintained, and regardless of the mobile nodes' absolute position or movement.

In both static DTLRC schemes, the mean throughput of the network steadily increases as the number of nodes increases. This is because the static schemes are dependent on the absolute position of the mobile nodes in the simulation area, and a higher number of nodes covers more area. Overall, the total amount of data exchanged in the simulations remains comparable between standard DTLRC and the static versions.

\subsubsection{Write Conflicts}

Tables 4 through 5 show the number of write conflicts for 10, 25, 50 and 100 nodes. The values are the "average", "maximum" and "minimum" total number of write conflicts per trial.

Table 4: Write Conflicts, 10 and 25 Nodes

\begin{tabular}{|c|c|c|c|c|c|c|}
\hline Parameter & \multicolumn{3}{|c|}{$\mathbf{1 0}$} & \multicolumn{3}{|c|}{$\mathbf{2 5}$} \\
\hline & $\mathrm{S}$ & $\mathrm{C}$ & $\mathrm{D}$ & $\mathrm{S}$ & $\mathrm{C}$ & $\mathrm{D}$ \\
\hline Average & 10.7 & 8.6 & 14.1 & 154.8 & 146.3 & 144.0 \\
Maximum & 42 & 34 & 48 & 412 & 268 & 339 \\
Minimum & 1 & 13 & 18 & 53 & 156 & 171 \\
\hline
\end{tabular}

Table 5: Write Conflicts, 50 and 100 Nodes

\begin{tabular}{|c|c|c|c|c|c|c|}
\hline Parameter & \multicolumn{3}{|c|}{$5 \mathbf{0}$} & \multicolumn{3}{c|}{$\mathbf{1 0 0}$} \\
\hline & $\mathrm{S}$ & $\mathrm{C}$ & $\mathrm{D}$ & $\mathrm{S}$ & $\mathrm{C}$ & $\mathrm{D}$ \\
\hline Average & 1235.5 & 1180.9 & 1410.5 & 11321.2 & 9065.1 & 13062.9 \\
Maximum & 1924 & 1667 & 6214 & 36488 & 11687 & 44888 \\
Minimum & 710 & 1221 & 1614 & 6931 & 9297 & 23568 \\
\hline
\end{tabular}

Here, connected DTLRC trends with the lowest number of write conflicts. This is expected, as less memory divergence can materialize when data is so efficiently spread across a large area.

Standard DTLRC provides comparable performance to connected DTLRC for 10,25 , and 50 nodes. The difference in the number of write conflicts becomes substantial with 100 nodes. This suggests that the rate of opportunistic contacts allows more nodes to receive data that is later conflicted. As discussed in $\S 7.1 .2$, the coherence of memory increases at the expense of resolving more write conflicts. 
Disconnected DTLRC trends with a higher number of write conflicts than either of the other systems, with exception of the 25 node trials. This is because isolated static nodes can accumulate large amounts of conflicting data, which would have been resolved earlier had the node had more regular contact with the rest of the network.

\subsubsection{Time to Convergence}

Tables 6 through 9 present the time to convergence results. The first row of these tables is the average number of unresolved write conflicts existing at the very end of the trial. The "average" and "minimum" are the average and minimum time to the convergence of a write to an index in hours (h), minutes (m) and seconds (s), respectively.

Table 6: Time to Convergence, 10 Nodes

\begin{tabular}{|c|c|c|c|}
\hline Parameter & \multicolumn{3}{|c|}{$\mathbf{1 0}$} \\
\hline & $\mathrm{S}$ & $\mathrm{C}$ & $\mathrm{D}$ \\
\hline UWCs & 0.6 & 1.4 & 1.0 \\
Average & $11 \mathrm{~m} 9.6 \mathrm{~s}$ & $1 \mathrm{~m} 7.4 \mathrm{~s}$ & $3 \mathrm{~m} 46.3 \mathrm{~s}$ \\
Maximum & $18 \mathrm{~m} 36.0 \mathrm{~s}$ & $1 \mathrm{~m} 11.1 \mathrm{~s}$ & $2 \mathrm{~m} 18.9 \mathrm{~s}$ \\
\hline
\end{tabular}

Table 7: Time to Convergence, 25 Nodes

\begin{tabular}{|c|c|c|c|}
\hline Parameter & \multicolumn{3}{|c|}{$\mathbf{2 5}$} \\
\hline & $\mathrm{S}$ & $\mathrm{C}$ & $\mathrm{D}$ \\
\hline UWCs & 5.7 & 6.3 & 3.5 \\
Average & $10 \mathrm{~m} 53.5 \mathrm{~s}$ & $5 \mathrm{~m} 32.3 \mathrm{~s}$ & $8 \mathrm{~m} 57.6 \mathrm{~s}$ \\
Maximum & $17 \mathrm{~m} 51.9 \mathrm{~s}$ & $14 \mathrm{~m} 21.1 \mathrm{~s}$ & $13 \mathrm{~m} 30 \mathrm{~s}$ \\
\hline
\end{tabular}

Table 8: Time to Convergence, 50 Nodes

\begin{tabular}{|c|c|c|c|}
\hline Parameter & \multicolumn{3}{|c|}{$\mathbf{5 0}$} \\
\hline & $\mathrm{S}$ & $\mathrm{C}$ & $\mathrm{D}$ \\
\hline UWCs & 22.5 & 22.8 & 9.7 \\
Average & $10 \mathrm{~m} 22.8 \mathrm{~s}$ & $11 \mathrm{~m} 34.3 \mathrm{~s}$ & $15 \mathrm{~m} 54.3 \mathrm{~s}$ \\
Maximum & $1 \mathrm{~h} 19 \mathrm{~m} 27.3 \mathrm{~s}$ & $1 \mathrm{~h} 30 \mathrm{~m} 11.1 \mathrm{~s}$ & $40 \mathrm{~m} 26.7 \mathrm{~s}$ \\
\hline
\end{tabular}

Table 9: Time to Convergence, 100 Nodes

\begin{tabular}{|c|c|c|c|}
\hline Parameter & \multicolumn{3}{|c|}{$\mathbf{1 0 0}$} \\
\hline & $\mathrm{S}$ & $\mathrm{C}$ & $\mathrm{D}$ \\
\hline UWCs & 85.9 & 91.4 & 47.9 \\
Average & $10 \mathrm{~m} 50.6 \mathrm{~s}$ & $20 \mathrm{~m} 31.7 \mathrm{~s}$ & $20 \mathrm{~m} 53.4 \mathrm{~s}$ \\
Maximum & $3 \mathrm{~h} 33 \mathrm{~m} 54 \mathrm{~s}$ & $3 \mathrm{~h} 59 \mathrm{~m} 14 \mathrm{~s}$ & $1 \mathrm{~h} 55 \mathrm{~m} 15.6 \mathrm{~s}$ \\
\hline
\end{tabular}


With standard DTLRC, the average TTC remains relatively constant regardless of the number of nodes in the network. This is another indication that the performance of standard DTLRC remains consistent, regardless of the number of nodes in the network.

The average TTC for both static variants increases with the number of mobile nodes. This is because a greater number of mobile nodes presents an elevated probability that any specific node will be outside the transmission radius of any of the available static nodes. In this case, the mobile node must simply wait until it travels to within a static node's range before its writes can be shared and inconsistencies in its memory be made coherent.

\section{Social Cache Simulation}

Social Cache simulations were performed with the same data sets generated for the DTLRC simulations. In HCMM, varying the number of nodes affects the mobility patterns of peers [18]. This being the case, a subset of four nodes which repeatedly met during the simulation were selected as the hosts for Social Cache.

The underlying connection model utilized was the same as discussed in $\S 6.4$. The following metrics were recorded:

- Write Conflicts $(W C)$ : The average total, average maximum and average minimum number of write conflicts per trial; and,

- Time to Fulfillment (TTF): The interval between a peer's request for the data at a specific index, and that request being fulfilled by the owner of that index. The average, maximum and minimum TTF were recorded.

\section{Social Cache Results}

Table 10 presents the Social Cache simulation results. The following times are presented in hours (h), minutes (m) and seconds (s). Presented "maximums" and "minimums" are absolute, not average.

Table 10: Time to Fulfillment Summary

\begin{tabular}{|c|c|c|c|c|}
\hline Parameter & $\mathbf{1 0}$ & $\mathbf{2 5}$ & $\mathbf{5 0}$ & $\mathbf{1 0 0}$ \\
\hline Average & $19 \mathrm{~m} 2 \mathrm{~s}$ & $20 \mathrm{~m} 11 \mathrm{~s}$ & $30 \mathrm{~m} \mathrm{20s}$ & $16 \mathrm{~m} 37 \mathrm{~s}$ \\
Maximum & $6 \mathrm{~h} 55 \mathrm{~m} 40 \mathrm{~s}$ & $5 \mathrm{~h} 15 \mathrm{~m} 35 \mathrm{~s}$ & $6 \mathrm{~h} 27 \mathrm{~m} \mathrm{44s}$ & $4 \mathrm{~h} 49 \mathrm{~m} 19 \mathrm{~s}$ \\
Minimum & $0 \mathrm{~s}$ & $0 \mathrm{~s}$ & $0 \mathrm{~s}$ & $0 \mathrm{~s}$ \\
\hline
\end{tabular}

In the worst case, requests for data were not filled within half the simulated time span. However, most requests for data were filled in less than half an hour. The minimum times for each simulated set were all 0 seconds, indicating that the requesting peer had an open connection with the source peer at the time the request was made.

The number of write conflicts recorded during the Social Cache experiments were trivial, often zero and never more than 10. 


\section{Conclusion}

This paper presents Delay Tolerant Lazy Release Consistency and Social Cache as methodologies for maintaining the consistency of shared data in opportunistic networks. Simulation results demonstrate that DTLRC and SC are viable technologies in a variety of scenarios where opportunistic contacts occur among wireless devices.

Future work on DTLRC and SC would include:

- Introduce additional conflict resolution protocols that allow shared data to reach a meaningful, consistent state while remaining generic enough to be utilized by an assortment of applications;

- Adapt DTLRC and SC for utilization across multiple hops with an ON;

- Explore potential issues and solutions relating to the security of DTLRC and SC; and,

- Implement fault tolerance should a node in a SC system be permanently disconnected from its peers.

The presented issues, as well as others, can increase an itinerant wireless device's ability to effectively collaborate and share content with peers.

\section{Acknowledgments}

This work was supported in part by the US National Science Foundation under grant CNS-0834493. Any opinions, findings, and conclusions or recommendations expressed in this paper are those of the authors and do not necessarily reflect the views of the National Science Foundation.

The authors would like to thank Chiara Boldrini and Andrea Passarella for use of the HCMM source code.

The authors would like to thank the anonymous reviewers for their constructive comments to improve the content and quality of presentation.

\section{References}

[1] W. Zhao, M. Ammar, E. Zegura, A message ferrying approach for data delivery in sparse mobile ad hoc networks, in: Proceedings of the 5th ACM International Symposium on Mobile Ad Hoc Networking and Computing, MobiHoc '04, ACM, New York, NY, USA, 2004, pp. 187 - 198.

[2] L. Pelusi, A. Passarella, M. Conti, Opportunistic networking: Data forwarding in disconnected mobile ad hoc networks 44 (11) (2006) $134-141$.

[3] S. Jain, K. Fall, R. Patra, Routing in a delay tolerant network, SIGCOMM Comput. Commun. Rev. 34 (2004) 145 - 158. 
[4] A. S. Tanenbaum, M. van Steen, Distributed Systems: Principles and Paradigms, 2 ed., Prentice Hall, Upper Saddle River, NJ, 2006.

[5] L. Lamport, How to make a multiprocessor computer that correctly executes multiprocess programs C-28 (9) (1979) 690 - 691.

[6] P. W. Hutto, M. Ahamad, Slow memory: Weakening consistency to enhance concurrency in distributed shared memories, in: Distributed Computing Systems, 1990. Proceedings., 10th International Conference on, 1990, pp. $302-309$.

[7] M. Conti, M. Kumar, Opportunities in opportunistic computing 43 (1) (2010) $42-50$.

[8] W. Stallings, Wireless Communications and Networks, 2 ed., Prentice Hall, Upper Saddle River, NJ, USA, 2005.

[9] A. Kshemkalyani, M. Singhal, Distributed Computing: Principles, Algorithms and Systems, Cambridge University Press, New York, NY, USA, 2008 .

[10] M. Wiesmann, F. Pedone, A. Schiper, B. Kemme, G. Alonso, Understanding replication in databases and distributed systems, in: Distributed Computing Systems, 2000. Proceedings. 20th International Conference on, 2000, pp. $464-474$.

[11] K. Gharachorloo, D. Lenoski, J. Laudon, P. Gibbons, A. Gupta, J. Hennessy, Memory consistency and event ordering in scalable shared-memory multiprocessors, in: Computer Architecture, 1990. Proceedings., 17th Annual International Symposium on, 1990, pp. $15-26$.

[12] C. Amza, A. Cox, S. Dwarkadas, P. Keleher, H. Lu, R. Rajamony, W. Yu, W. Zwaenepoel, Treadmarks: shared memory computing on networks of workstations, Computer 29 (2) (1996) $18-28$.

[13] P. Keleher, Lazy release consistency for distributed shared memory, Ph.D. thesis, Rice University, Houston, Texas, USA (1994).

[14] W.-Y. Liang, Y.-M. Hsieh, Z.-Y. Lyu, Design of a dynamic distributed mobile computing environment, in: Parallel and Distributed Systems, 2007 International Conference on, Vol. 2, 2007, pp. 1-8.

[15] D. Stall, L. Stall, Antiqueweekend.com - texas antique weekend shows \& events - round top - warrenton - fayette county, tx., accessed: February 6th, 2013.

URL AntiqueWeekend.com

[16] U.S. Census Bureau, 2010 census, U.S. Department of Commerce (Feb. 2011). 
[17] T. Kelly, Combinatorial auctions and knapsack problems, Proceedings of the Third International Joint Conference on Autonomous Agents and Multiagent Systems (2004) 1278-1279.

[18] C. Boldrini, A. Passarella, HCMM: Modelling spatial and temporal properties of human mobility driven by users social relationships, Computer Communications 33 (9) (2010) 1056-1074.

[19] C. Boldrini, M. Conti, F. Delmastro, A. Passarella, Context- and socialaware middleware for opportunistic networks, Journal of Network and Computer Applications 33 (5) (2010) 525-541.

[20] G. Chakraborty, K. Naik, D. Chakraborty, N. Shiratori, D. Wei, Analysis of the bluetooth device discovery protocol, Wirel. Netw. 16 (2) (2010) 421436.

[21] Bluetooth Special Interest Group (SIG), Bluetooth Specification Version $2.1+$ EDR [vol 0], https://www.bluetooth.org/docman/handlers/downloaddoc. ashx?doc_id=241363 (Jul. 2007). 